\title{
LXII. On the geometrical interpretation of quaternions
}

\section{W.F. Donkin M.A.}

To cite this article: W.F. Donkin M.A. (1850) LXII. On the geometrical interpretation of quaternions, Philosophical Magazine Series 3, 36:246, 489-503, DOI:

10.1080/14786445008646538

To link to this article: http://dx.doi.org/10.1080/14786445008646538

册 Published online: 30 Apr 2009.

Submit your article to this journal $₫$

Џ Article views: 4

Q View related articles $₫$ 


\title{
PHILOSOPHICAL MAGAZINE AND JOURNAL OF SCIENCE.
}

\author{
SUPPLEMENT ro VOL. XXXVI. THIRD SERIES.
}

LXII. On the Geometrical Interpretation of Quaternions. By W. F. Donkin, M.A., \&c., Savilian Professor of Astronomy in the University of Oxford*.

T $N$ a recent paper in this Journal, I gave two examples of 1 the application of quaternions to geometrical problems, in which 1 made use of a system of interpretation different from that usually employed by Sir W. Hamilton. This system it is my present object to explain. I am not at present prepared to offer an opinion as to its practical advantages or disadvantages. It is here proposed as possessing a certain theoretic interest, arising from the fact that it represents the method of quaternions as a natural (or rather the natural) extension to tridimensional space of the usual geometrical interpretations of symbolical algebra. The general principles employed in the investigation have no pretensions to novelty. The view here taken of symbolical algebra, and of its interpretation in plane geometry, is substantially the same as that advocated by the late Mr. Gregory (in the fourteenth volume of the Edinburgh Philosophical Transactions, and in several papers in the Cambridge Mathematical Journal); and the extension to geometry of three dimensions rests upon principles which will be found, I believe, to agree essentially, as far as they go, with those laid down by Sir W. Hamilton in his papers on symbolical geometry in the last-named periodical. In what follows, then, no further reference will in general be made to previous writers. Moreover, to avoid the use of frequent qualifying phrases, propositions will be stated dogmatically which only claim to be accepted as belonging to a consistent system, not as belonging to the only consistent or useful system.

General symbolical algebra is a calculus of operations. All the symbols employed, including those arithmetical symbols which occur, represent or indicate operations. The subject

* Communicated by the Author.

Phil. Mag. S. 3. No. 246, Suppl. Vol. 36. 
of operation, or (to adopt Sir W. Hamilton's convenient term) the operand, is in general not represented by any symbol at all, but is to be understood. Thus the symbol $a b$ represents the successive performance of the operations $b, a$ upon the operand. Neither operations nor operand are defined, further than definition is involved in the assumed laws of the combination of symbols. If we assume $a b=b a$, we limit to a certain extent the choice of meanings for the operations $a$ and $b$; and if we assume that the arithmetical equation $m+n=s$ shall be true in symbolical algebra (where $m$ and $n$ are absolute numbers, and $s$ their sum), we limit the choice of the operand: for let it be a ; then the above equation signifies $m \mathbf{a}+n a=s a$, and the meaning of a must be such that this may be capable of an intelligible and consistent interpretation. It is not necessary for our present purpose to attempt a complete discussion of the laws of the usual algebraical symbols. The following remarks, however, may serve to elucidate the processes to be explained hereafter.

The symbols + and - are used in algebra in two essentially different ways. First, they are used, as in arithmetic, to indicate addition and subtraction. Thus $a+b$ denotes a certain resultant formed by separately performing the operations $a$ and $b$ on the operand, and adding the result of the latter operation to that of the former. The meaning of addition cannot be defined till the nature of the operand is determined. The same may be said of $a-b$, mutatis mutandis. The equations

$$
a+(b+c)=a+b+c, \& c .
$$

suggested by arithmetic as convenient assumptions, suggest in their turn further assumptions; namely, that if + and should ever be used as true symbols of operation, so that + a should denote the result of performing the operation + upon the operand a, and $+-a$ should denote the performance of the successive operations $a,-,+$ upon an operand (not expressed), then the laws of their combinations should be

$$
++=--=+, \quad+-=-+=- \text {. }
$$

Such combinations do not really occur at all in the first use of these symbols. The expression $a-(b-c)$ only suggests, but does not really contain the combination - -

We now come to the second way in which + and - are used, namely, as true symbols of operation, defined by the laws of combination just stated.

Let us for the present distinguish this use of the symbols by inclosing them in brackets. Then the following equations 
are well known, viz.

$$
(+)^{n}=(+), \quad(-)^{2 n}=(+), \quad(-)^{2 n-1}=(-),
$$

where $n$ is an integer. But it is to be remembered, that, unless $n=1$, these equations do not express identity, but only equivalence. $(-)^{2}=(+)$ is an identical equation; but $(+)^{2}=(+)$ does not mean that $(t)^{2}$ is the same operation as $(t)$, but only that it produces the same result when performed on the operand. Thus $(+)^{2}=(t)$ is only true in the same sense in which $(+)=1$ is true. And this brings us to a point of considerable importance, namely, the twofold use of the symbol 1 in algebra, as representing an operation on the one hand, or a concrete quantity or operand on the other. Considered in the former light, 1 represents the operation of taking the operand as it is; considered in the latter, it represents a concrete unit taken as a subject of operation. In the former case $(+) 1$ and $(-) 1$ may be used as synonymous with $(+)$ and $(-)$, and $\sqrt{(+) 1}, \sqrt{(-)^{1}}$ as synonymous with $(+)^{\frac{1}{2}},(-)^{\frac{1}{2}}$. In the latter case

$$
((+) 1)^{2},((-) 1)^{2}, \sqrt{(+) 1}, \sqrt{(-) 1}
$$

are all equally nnmeaning, just as

$$
((+) \mathfrak{E 1})^{2}, \quad \sqrt{(-) \mathfrak{E} 1,} \text { \&c. }
$$

are equally unmeaning. It appears to me that a great deal of confusion has arisen from neglecting this distinction. At all events $I$ intend to preserve it strictly in the present paper, in which all the symbols will represent operations, and never concrete quantities, unless that be expressly stated. Thus, if $x$ be one of the coordinates of a point in space, $x$ represents, strictly speaking, in any equation in which it may occur, not a line, nor a number, but the operation of multiplying the operand (which is understood throughout) by the same number by which the linear unit must be multiplied in order to produce a line equal to the distance of the point in question from the plane of $y z$. If this be well understood, there is no objection to speaking of $x$, for convenience, as if it really represented such a line.

To return, however, to the symbols + and - , the equations

$$
a+(+) b=a-(-) b=a+b, \quad a-(+) b=a+(-) b=a-b,
$$

which rest on grounds that need not be here examined, justify us in omitting the use of brackets to save trouble, though it would perhaps be theoretically desirable to have some equivalent mark of distinction.

'The principles of the usual interpretation of symbolical $2 \mathrm{~K} 2$ 
algebra in plane geometry are now generally understood and recognized. There are some important remarks, however, to be made with reference to what follows. The operand, in this system of interpretation, is always a directed line, that is, a straight line in a determinate direction, and with a determinate beginning and end. It is convenient for our present purpose to assume that all the lines considered shall have their beginnings at the same point or origin. The operand, then, is a radius vector drawn from a determinate origin in a determinate plane. The symbol + denotes the operation of turning this line round the origin through a whole revolution, in a determinate sense, which we will assume to be contrary to that of the motion of the hands of a watch. It is especially important to observe, that + does not represent the operation of placing the line in a determinate divection (such as that of a given fixed axis), but the operation of turning it round from the direction it had at first (which may be any whatever) till it comes into the same direction again. Then $(+)^{\frac{1}{2}}$, or - , represents a semi-revolution; $(-)^{\frac{1}{2}}$, or, as it is commonly written, $\sqrt{-1}$, a quarter of a revolution; and so on. And if $a$ be a numerical symbol, then $(+)^{\alpha} a$ represents the compound operation of altering the length of the operand line in the ratio of $a$ to 1 , and also turning it round through an angle equal to $2 \alpha \pi$.

The addition and subtraction of directed lines is to be performed, as is well known, according to the rules for the composition of forces. Thus, if $a, b$ be two radii vectores, $a+b$ represents another radius vector, namely the diagonal (drawn from the origin) of the parallelogram constructed on them; and $a-b$ represents a third radius vector, namely the diagonal (from the origin) of the parallelogram of which two sides are $a$, and a line equal to $b$, but in the opposite direction. The symbol $\cos \theta+\sqrt{-1} \cdot \sin \theta$ represents a compound operaration, namely (1) multiplying the operand by $\cos \theta$ without altering its direction; (2) multiplying it by $\sin \theta$ and turning it through a right angle; (3) adding together the two lines so obtained, or taking the diagonal of the parallelogram described upon them; which diagonal is obviously equal in length to the original operand, but inclined to it at an angle $\theta$, so that $\cos \theta+\sqrt{-1} \cdot \sin \theta$ represents the operation of turning through an angle $\theta$, and is equivalent to $(+)^{\alpha}$, if $\theta=2 \alpha \pi$. Nothing depends, as was before observed, on the direction of the operand line; but in interpreting such expressions as $p+q$, where $p, q$ are any symbols of operation, of course it is assumed that both refer to the same operand. In plane geometry, the 
disection of the operand line is in general completely arbitrary in its own plane; but this is not generally true when it is a line in space not confined to a determinate plane. I now proceed to consider the application of algebraical symbols to this case.

If we assume the symbol $+r$ to represent the operation of turning a line through a complete revolution in a plane perpendicular to a determinate axis $r$, drawn from the origin, then $\left(+_{r}\right)^{a}$ will represent the operation of turning through an angle $2 a \pi$; and we may use $-r$ as a symbol equivalent to $(+r)^{\frac{1}{2}}$, denoting a semi-revolution. So far as rotations in this one plane are concerned, all that has been said of $(t)$ and $(-)$ in plane geometry will apply to $t_{r}$ and $-{ }_{r}$. I will add, however, one remark, which may be useful to readers (if this paper should meet with such) to whom the subject is new. If we admit (which, however, is not necessary) the idea of negative angles, then, $\theta$ representing an angle described by a determinate rotation, $-\theta$ represents an equal angle described by a contrary rotation. But if $q$ represent the operation of describing the angle $\theta$, or the rotation by which it is described, then the operation of describing $-\theta$ is represented, not by $-q$, but by $\frac{1}{q}$ or $q^{-1}$. The equation $\theta+(-\theta)=0$ means the same thing as $q^{-1} q=1$. The former expresses that the angular distance between the first and last positions of the describing line is 0 ; the latter, that if we turn a line through any angle and then back again, the result is equivalent to simply taking it as it is, which, considered as an operation, is represented by the symbol 1 . To justify the assertion that the consideration of negative angles is not necessary, it will be sufficient to observe that an angle which is negative when considered as described about one axis, is positive if it be considered as described about an opposite axis. But a complete discussion of this point would involve an examination of the theory of indices, and would lead us too far from our immediate subject, to which I return.

For convenience let $q$ be put for $\left(+_{r}\right)^{\alpha}$, so that $q$ is a symbol of rotation, and represents the operation of turning the operand line through an angle $\theta(=2 \alpha \pi)$ in a plane perpendicular to a given line $r$ (whose length is immaterial) drawn from the origin; the direction of rotation, moreover, being such that the line $r$ shall be its positive or north axis. The initial position of the operand must be in the plane of the rotation, otherwise the operation could not be performed upon it: but so far as this one operation is concerned, it may be in any 
part of the plane; and the symbol $q$ represents, indifferently, rotation through an angle $\theta$ in any part of the plane.

Now let $q^{\prime}=\left(+r^{\prime}\right)^{\beta}$ represent in like manner the operation of turning through an angle $\theta^{\prime}=2 \beta \pi$ in another plane whose axis is $r^{\prime}$. The two operations $q, q^{\prime}$ cannot in general be successively performed upon the same operand line. In order that this may be possible, it is necessary to place it at first in the plane of the first rotation $g$, and in such a direction that this first rotation shall bring it into the line of intersection of the two planes, whereby the second rotation becomes possible.

Let $q^{\prime \prime}$ represent the single rotation which would have brought the line from its initial position into that final position in which it is placed by the successive rotations $q, q^{\prime}$; we have then the symbolic equation (or rather equivalence) $q^{\prime} q=q^{\prime \prime}$. 'The successive rotations $q, q^{\prime}$ are (not identical with, but) equivalent in effect to the single rotation $q^{\prime \prime}$. If now a third rotation $q^{\prime \prime \prime}$ is to be performed, the rotation $q^{\prime \prime}$ must (if necessary) be transported in its own plane so as to make the compound operation $q^{\prime \prime \prime} q^{\prime \prime}$ possible, just as the first rotation $q$ was transported so as to make $q^{\prime} q$ possible. These conventions enable us to assign a determinate rotation in a determinate plane as the result (or product) of any number of given successive rotations in given planes. But it is obvious that no useful method of calculation could be based upon such assumptions, unless we could prove (as we can prove) that the associative principle holds good with respect to the product of rotations; that is, that $\left(q^{\prime \prime} q^{\prime}\right) q=q^{\prime \prime}\left(q^{\prime} q\right)$.

Let us now adopt a method of representing rotations (in words or by actual diagrams) similar to that en ployed in the previous paper. Conceive a sphere with arbitrary radius to be described about the origin; and, $A B$ being any arc of a great circle on this sphere, let "the rotation $A B^{3}$ signify that rotation of a radius vector which would cause its intersection with the sphere to describe the arc AB (from $\mathrm{A}$ to $\mathrm{B}$ ), or any equal and similarly described arc in the same great circle. [It must be carefully borne in mind, that we are now concerned with the rotations of lines, and not, as in the former paper, of solids].

Let $A B C$ be any spherical triangle, and let the rotations $\mathrm{AB}, \mathrm{BC}, \mathrm{AC}$ be represented as above by $q, q^{\prime}, q^{\prime \prime}$; we have then $q^{\prime} q=q^{\prime \prime}$. It is easy to see that $q q^{\prime}$ does not represent the same thing as $q^{\prime} q$, and also to see what it does represent. For producing $A B$ to $A^{\prime}$ and $C B$ to $C^{\prime}$, making $B^{\prime}=A B$, $\mathrm{BC}^{\prime}=\mathrm{CB}$, and joining $\mathrm{C}^{\prime} \mathrm{A}^{\prime}$, we see that $q q^{\prime}$ represents the successive rotations $\mathrm{C}^{\prime} \mathrm{B}, \mathrm{BA}^{\prime}$, the effect of which is the same as that of $\mathrm{C}^{\prime} \mathrm{A}^{\prime}$. If, then, we denote th s last rotation by $q_{\mu}$, 
we have $q q^{\prime}=q_{\mu l}$; and since $\mathrm{C}^{\prime} \mathrm{A}^{\prime}$ is equal to $\mathrm{AC}$, we see also that $q q^{\prime}, q^{\prime} q$ represent equal rotations, but generally in different planes; these planes, however, being equally inclined to the planes of $q, q^{\prime}$.

Consider now in particular the case in which $\mathrm{AB}, \mathrm{BC}$ are both quadrants. Then it is obvious that $\mathrm{C}^{\prime} \mathrm{A}^{\prime}$ is in the same great circle with $\mathrm{AC}$, so that if $q^{\prime \prime}$ represent the rotation $\mathrm{AC}$, $\frac{1}{q^{\prime \prime}}$ will represent the rotation $C^{\prime} A^{\prime}$, and we have therefore, in this case, $q q^{\prime}=\left(q^{\prime} q\right)^{-1}$. Now let $q$, represent (not $\mathrm{AC}$ but) $\mathrm{CA}$, so that $q, q, q$, represent the rotations by which the sides of the triangle would be described in a cyclic order; then we shall have, for any triangle,

$$
q^{\prime} q=\frac{1}{q_{l}}, \quad q q_{i}=\frac{1}{q^{\prime}}, \quad q_{i} q^{\prime}=\frac{1}{q} ;
$$

and if we further suppose all the sides of the triangle to be quadrants, we shall also have, as we have just seen,

$$
q q^{\prime}=q_{1}, \quad q_{l} q=q^{\prime}, \quad q^{\prime} q_{l}=q .
$$

Before we proceed further, there is one important remark to be made with respect to the symbol $\left(+_{r}\right)$, or $--_{r}$; namely, that the effect of the rotation denoted by it, is, regard being had to the conventions above established, independent of the direction of the axis $r$; since a semi-revolution, in any plane in which it is possible, merely reverses the direction of the operand line. We may therefore in all cases substitute the general symbol - for $-r$, and write $\left(+_{r}\right)^{\frac{d}{2}}=-$. To illustrate this further, let $q$ represent any rotation $\mathrm{AB}$, and let us examine the meaning of the product $-{ }_{r} q$. The arc $\mathrm{AB}$ must be so placed in its own plane that its end $B$ shall be at the intersection of that plane with the plane of the rotation $-r_{r}$; then the operand line must first describe $A B$, and then half a circumference in the latter plane, after which it will obviously be again in the same great circle with $\mathrm{AB}$, cutting the,sphere in the point opposite to $B$. Now the same effect would have been produced by a single rotation in the plane of the rotation $q$, through an angle $\theta+\pi$ described positively, or $\pi-\theta$ described negatively, $\theta$ being the angle of the rotation $q$. If, then, $q=\left(+_{r}\right)^{\alpha}$, we have

$$
\left(-{ }_{r}\right) q=\left(+{ }_{r}\right)^{\alpha \pm \frac{1}{2}}
$$

in which we may henceforth drop the subscript $r$, and write simply $-q$ on the first side.

It is still more obvious that similar remarks apply to the case of the symbol $+_{r}$; or that we may always drop the sub- 
script, and use + simply, to denote a whole revolution in any plane. But there are no other values of $\alpha$ besides these two of $\alpha=1, \alpha=\frac{1}{2}$, and their multiples, for which the effect of the rotation $\left(+_{r}\right)^{\alpha}$ is independent of the direction of $r$. It is also to be noticed, that these are the only cases in which the symbol $\left(+_{r}\right)^{\alpha}$ is commutative with respect to all other symbols of rotation. 'The reader will easily see, that, on the principles we have admitted, $\left(+_{r}\right)^{\frac{1}{2}} q$ is equivalent to $q\left(+_{r}\right)^{\frac{1}{2}}$. Either will be henceforth denoted by $-q$.

Now let there be three fixed rectangular axes meeting the sphere in $X, Y, Z$, and so arranged that $X$ is the north pole of the rolation $\mathrm{YZ}$. Let us for convenience assume three fixed symbols, $i, j, k$, to represent the three rotations $\mathrm{YZ}$, $\mathrm{ZX}, \mathrm{XY}$; that is, quadrantal rotations respectively in (any parts of) the three planes of $y z, z x, x y$. According to our previous notation, we might write

$$
i=\left(+_{x}\right)^{\frac{1}{3}}, \quad j=\left(+_{y}\right)^{\frac{1}{4}}, \quad k=\left(+_{z}\right)^{\frac{4}{4}} \text {; }
$$

but we shall not have much further occasion for it.

The conclusions obtained above respecting quadrantal triangles give the equations

$$
j k=i, \quad k i=j, \quad i j=k, \quad k j=\frac{1}{i}, \quad i k=\frac{1}{j}, \quad j i=\frac{1}{k} ;
$$

of which the three last can now be written

For we have

$$
k j=-i, \quad i k=-j, \quad j i=-k \text {. }
$$

$$
i^{-1}=\left(+{ }_{x}\right)^{-\frac{1}{4}}=\left(+_{x}\right)\left(+x_{x}\right)^{-\frac{1}{4}}=\left(++_{x}\right)^{\frac{3}{4}}=\left(+{ }_{x}\right)^{\frac{1}{4}}\left(+_{x}\right)^{k}=-{ }_{x} i ;
$$

and we have seen that $-{ }_{\star}$ may be replaced by - , so that we get $i^{-1}=-i$, and, in like manner,

$$
j^{-1}=-j, \quad k^{-1}=-k .
$$

Also we have

and similarly,

$$
i^{2}=\left(+{ }_{x}\right)^{\frac{1}{2}}=-
$$

$$
j^{2}=-y, \quad k^{2}=-{ }_{z} ;
$$

which may now be written

$$
i^{2}=\jmath^{2}=k^{2}=-;
$$

and, to conform to common notation, we may put -1 instead of - . In all these cases it is to be recollected that the symbol $=$ denotes, not identity of operations, but equivalence of results. 
The associative principle, so far as the products of the quadrantal rotations represented by $i, j, k$ are concerned, is now established, since

$$
(i j) k=k^{2}=-1, \quad i(j k)=i^{2}=-1,8 \mathrm{c} .
$$

Hitherto we have been considering the use of the symbol $t_{r}$ as representing rotation. We must now proceed to examine the principles on which the symbols + and - are to be employed in the present system, as indicating the addition and subtraction of directed lines.

Let $q, q^{\prime}$ denote any two rotations, and $a, a^{\prime}$ two numbers. What is the interpretation of $a q+a^{\prime} q^{\prime}$ ? In the first place, $a q$ represents the compound operation of turning the operand line through the rotation $q$, and altering its length in the ratio of $a$ to 1 ; and $a^{\prime} q^{\prime}$ has a similar meaning. Next, in order that $a q, a^{\prime} q^{\prime}$ may both refer to the same operand line, that line must at first coincide with the intersection of the planes of the two rotations $q, q^{\prime}$. Let a be the operand so placed; then $a q a, a^{\prime} q^{\prime} \mathbf{a}$ represent two lines whose lengths are $a \mathrm{a}, a^{\prime} \dot{a}$, and whose directions are determined by the rotations $q, q^{\prime}$. 'Their sum $a q a+a^{\prime} q^{\prime}$ a represents the diagonal of the parallelogram constructed upon them. Let $a^{\prime \prime} a$ be the length of this diagonal, and $q^{\prime \prime}$ the rotation which would bring the original operand into coincidence with it; then we have

$$
a q \mathrm{a}+a^{\prime} q^{\prime} \mathrm{a}=a^{\prime \prime} q^{\prime \prime} \mathrm{a},
$$

or onitting the symbol of the operand,

$$
a q+a^{\prime} q^{\prime}=a^{\prime \prime} q^{\prime \prime}
$$

Thus the complex symbol of operation, $a q+a^{\prime} q^{\prime}$, represents a single determinate rotation combined with a determinate alteration of length. It is easy to illustrate this by a diagram, thus:-

Let $\mathrm{ABC}$ be any spherical triangle, and let $q, q^{\prime}$ represent the rotations $\mathrm{AB}, \mathrm{AC}$. Then $\left(a q+a^{\prime} q^{\prime}\right)$ a represents the diagonal of a parallelogram constructed on two radii vectores which cut the sphere in $B$ and $C$, and whose lengths are $a$, $a^{\prime}$ a. This diagonal will cut the sphere in a point $\mathrm{D}$ of the arc $\mathrm{BC}$, such that

$$
\sin \mathrm{BD}: \sin \mathrm{CD}:: a^{\prime}: a \text {. }
$$

Join $\mathrm{AD}$; then, in the equation $a q+a^{\prime} q^{\prime}=a^{\prime \prime} q^{\prime \prime}$, we have obviously

$$
a^{\prime \prime 2}=a^{2}+a^{\prime 2}+2 a a^{\prime} \cos \mathrm{BC},
$$

and $q^{\prime \prime}$ is the symbol of the determinate rotation AD.

The expression $a+a^{\prime} q^{\prime}$, which is merely a particular case of the above, is easily interpreted in the same way. The ope- 
rand a must first be in the plane of the rotation $q^{\prime}$. The operation $a$ merely alters its length into $a \mathrm{a}$; the operation $a^{\prime} q^{\prime}$ alters its length into $a^{\prime}$ a, and also alters its direction. Then $\left(a+a^{\prime} q^{\prime}\right)$ a is the diagonal of the parallelogram constructed on the two lines represented by $a \mathfrak{a}, a^{\prime} q^{\prime} a$; and if $a^{\prime \prime} a$ be the length of this diagonal, and $q^{\prime \prime}$ the rotation which would bring the operand into coincidence with it, we have, as before,

The expression

$$
a+a^{\prime} q^{\prime}=a^{\prime \prime} q^{\prime \prime} \text {. }
$$

$$
a q+a^{\prime} q^{\prime}+a^{\prime \prime} q^{\prime \prime}
$$

in which $q, q^{\prime}, q^{\prime \prime}$ represent $a n y$ three rotations, and $a, a^{\prime}, a^{\prime \prime}$ any three numbers, cannot generally be interpreted without the help of conventions similar to those adopted in interpreting products; because unless the planes of the three rotations happen to intersect in the same line, it is impossible so to place the operand that each operation can be performed on it. But we have seen that $a q+a^{\prime} q^{\prime}$ is equivalent to the symbol of a single determinate rotation in a determinate plane, combined with a determinate alteration of length. Suppose, therefore,

$$
a q+a^{\prime} q^{\prime}=a_{i} q .
$$

Then we may transport the rotation $g_{1}$ in its own plane, until its origin coincides with the line of intersection of that plane with the plane of the rotation $q^{\prime \prime}$, so that $a_{1} q_{1}+a^{\prime \prime} q^{\prime \prime}$ may be interpretable in the same way as $a q+a^{\prime} q^{\prime}$. 'Thus we shall finally arrive at a single determinate rotation, with a determinate alteration of length, as the interpretation of

$$
\left(a q+a^{\prime} q^{\prime}\right)+a^{\prime \prime} q^{\prime \prime} \text {. }
$$

The usefulness of the system will depend upon our being able to show (as we shall easily do) that the associative principle holds good in addition, or that

$$
\left(a q+a^{\prime} q^{\prime}\right)+a^{\prime \prime} q^{\prime \prime}=a q+\left(a^{\prime} q^{\prime}+a^{\prime \prime} q^{\prime \prime}\right)=\left(a q+a^{\prime \prime} q^{\prime \prime}\right)+a^{\prime} q^{\prime},
$$

which will justify us in omitting the brackets.

The case of subtraction is evidently included in that of addition, since the sign -, preceding any symbol of rotation, may be changed into $t$, provided we increase the angle of rotation by two right angles.

We are now in a position to prove the following fundamental proposition :-

Let $i, j, k$ represent, as above explained, quadrantal rotations whose positive poles are respectively $\mathrm{X}, \mathrm{Y}, \mathrm{Z}$, and let $l, m, n$ be the direction cosines of any line $r$ from the origin. Then $i l+j m+k n$ represents a quadrantal rotation whose po- 
sitive axis is $r$; or, according to our previous notation,

If the condition

$$
i l+j m+k n=\left(+{ }_{r}\right)^{4} \text {. }
$$

$$
l^{2}+m^{2}+n^{2}=1
$$

did not subsist, then, putting

we should have

$$
\sqrt{l^{2}+m^{2}+n^{2}}=\mu
$$

$$
i l+j m+k n=\left(+{ }_{r}\right)^{\frac{1}{4}} \mu,
$$

representing the compound operation of turning the operand through a right angle in the plane perpendicular to $r$, and altering its length in the ratio of $\mu$ to 1 , the direction cosines of $r$ being proportional to $l, m, n$.

The proof of this is very simple, but can hardly be given without a diagram. In the figure (of which all the lines represent ares of great circles), let $X$, $\mathrm{Y}, \boldsymbol{Z}$ be the positive $\mathrm{X}$ poles of the rotations $i, j, k$, or the points in which the positive axes of $x, y, z$ cut the sphere; and let $X^{\prime}$, $\mathcal{Z}$ be diametrically opposite to $\mathrm{X}, \mathrm{Z}$.

Now to interpret

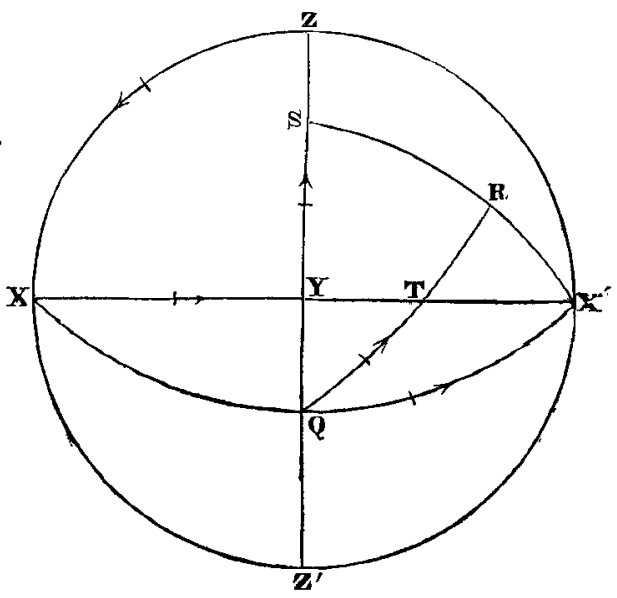
$m j+n k$, take $\mathrm{XZ}$ and $\mathrm{XY}$ to represent the rotations $j, k$; then taking $Q$ in $Y Z$, so that

$$
\sin \mathrm{YQ}: \sin Q Z^{\prime}:: n: n,
$$

and joining $X Q$, we have evidently, by the principles established above,

$$
m j+n k=\sqrt{m^{2}+n^{2}} \cdot q,
$$

where $q$ represents the quadrantal rotation XQ. Next, to interpret

$$
\sqrt{m^{2}+n^{2}} \cdot q+l i
$$

transport the rotation $q$ in its own plane to $\mathbf{Q X}$, so that its origin may be in the plane of the rotation $i$. Take QS a quadrant, and join $\mathrm{SX}^{\prime}$. Take $\mathrm{R}$ in $\mathrm{SX}^{\prime}$ so that

$$
\sin \mathrm{SR}: \sin \mathrm{RX}^{\prime}:: \quad \sqrt{m^{2}+n^{2}}: l \text {, }
$$


and join $\mathrm{QR}$. Then, by the same principles, we have

$$
\sqrt{m^{2}+n^{2} \cdot} q+l i=\sqrt{l^{2}+m^{2}+n^{2}} \cdot q^{\prime} \text {, }
$$

where $q^{\prime}$ represents the quadrantal rotation $Q R$, If wo suppose

$$
l^{2}+m^{2}+n^{2}=1,
$$

then what we have just proved is that

$$
i l+(j m+k n)
$$

represents the quadrantal rotation $Q R$, without alteration of the length of the operand.

Let $\mathrm{QR}$ cut $\mathrm{YX}^{\prime}$ in $\mathrm{T}$. Then in the right-angled triangle QY'T the preceding conditions give easily

$$
\cos \mathrm{YQT}=l, \quad \cos \mathrm{YQ}=\frac{n}{\sqrt{m^{2}+n^{2}}},
$$

whence, by Napier's rules,

$$
\cos \mathrm{YTQ}=n \text {. }
$$

Thus $l$ and $n$ are the cosines of the angles which the plane of QR makes with the planes of $y z, x y$; whence it follows that $m$ is the cosine of the angle which it makes with the plane of $a z$. In other words, $l, m, n$ are the direction cosines of the axis of the rotation $Q R$; and an examination of the figure constructed for any particular case, shows that they belong to the positive axis of that rotation.

We have thus interpreted the expression $i l+(j m+k n)$; and the symmetry of the result shows that the interpretation of $(i l+j m)+k n$ or of $(i l+k n)+j m$ would have conducted us to the same conclusion. Thus the associative principle of addition is established so far as quadrantal rotations are concerned, and we may write $i l+j m+k n$ without brackets.

Consider now the expression

$$
\cos \theta+\sin \theta(i l+j m+k n) \text {. }
$$

The first term represents the operation of altering the length of the operand in the ratio of $\cos \theta$ to 1 ; and the second, the compound operation of altering its length in the ratio of $\sin \theta$ to $l$, and turning it through a right angle in a determinate plane. Applying, therefore, the principles explained above with reference to the interpretation of such expressions as $a+a^{\prime} q^{\prime}$, we see that the complex symbol now under consideration represents the operation of turning the operand, without altering its length, through an angle $\theta$, in a plane perpendicular to the axis whose direction cosines are $l, m, n$. Let $r$ denote such an axis; then we may write

$$
\cos \theta+\sin \theta(i l+j m+k n)=(+,)^{\alpha},
$$


where

The quaternion

$$
\alpha=\frac{\theta}{2 \pi} .
$$

$$
w+i x+j y+k z
$$

is always reducible to the form

where

$$
\mu(\cos \theta+\sin \theta(i l+j m+k n)),
$$

by the assumptions

$$
l^{2}+m^{2}+n^{2}=1
$$

$\mu=\sqrt{w^{2}+x^{2}+y^{2}+z^{2}}, r=\sqrt{x^{2}+y^{2}+z^{2}}, \mu \sin \theta=r, \mu \cos \theta=w$,

$$
\frac{l}{x}=\frac{m}{y}=\frac{n}{z}=\frac{1}{r}
$$

and therefore it represents a rotation, combined with an alteration of length in the ratio of $\mu$ to 1 .

It must be observed, however, that we have so far only interpreted the expression

$$
w+(i x+j y+h z)
$$

and that we are not at liberty to remove the brackets without first establishing the associative principle of addition with respect to symbols of alteration of length, such as $w$, and symbols of alteration of length combined with quadrantal rotation, such as $i x, \& c$. But this is very easily done, and as easily for any rotations as for quadrantal.

Let $\mathrm{ABC}$ be any spherical triangle, and let $q, q^{\prime}$ represent the rotations $\mathrm{AB}, \mathrm{AC}$. Let $a, b, c$ be numerical quantities. Then the equations

$$
(a+b q)+c q^{\prime}=a+\left(b q+c q^{\prime}\right)=\left(a+c q^{\prime}\right)+b q,
$$

are easily seen to express, that if a parallelepiped be constructed with three edges coinciling in direction with the radii drawn to the points $\mathrm{A}, \mathrm{B}, \mathrm{C}$, and proportional in length respectively to $a, b, c$, then the diagonal of the parallelopiped may be obtained indifferently by taking the sum of any one of these edges and the diagonal of the parallelogram constructed on the other two.

We have now established* all that is necessary to give demonstrative force to geometrical conclusions deduced from algebraical calculation with quaternions. This paper has,

* The geometrical proof of the distributive property, expressed by such equations as

$$
i(a+j b)=i a+k b \text {, }
$$

$\& c_{\text {, }}$ is so easy and obvious, that $I$ leave it to the reader. 
however, already extended to such a length, that I will only give one simple instance of such reasoning.

Let $\mathrm{ABC}$ be any spherical triangle, and $q, q^{\prime}, q^{\prime \prime}$ represent the rotations $A B, B C, A C$. Then we have, as before explained, $q^{\prime \prime}=q^{\prime} q . \quad$ But if we put $\mathrm{AB}=\theta, B \mathrm{C}=\theta^{\prime}$, and call $l, m, n, l^{\prime}, m^{\prime}, n^{\prime}$ the direction cosines of the positive poles of $A B, B C$, then we have seen that

$$
q=\cos \theta+\sin \theta(i l+j m+k n),
$$

and $q^{\prime}$ is similarly composed of accented letters; hence

$$
\begin{aligned}
q^{\prime} q= & \cos \theta \cos \theta^{\prime}-\sin \theta \sin \theta^{\prime}\left(l l^{\prime}+m m^{\prime}+n n^{\prime}\right) \\
& +i\left\{l \sin \theta \cos \theta^{\prime}+l^{\prime} \sin \theta^{\prime} \cos \theta+\left(m^{\prime} n-m n^{\prime}\right) \sin \theta \sin \theta^{\prime}\right\} \\
& +j\left\{n \sin \theta \cos \theta^{\prime}+m^{\prime} \sin \theta^{\prime} \cos \theta+\left(n^{\prime} l-n l^{\prime}\right) \sin \theta \sin \theta^{\prime}\right\} \\
& +k\left\{n \sin \theta \cos \theta^{\prime}+n^{\prime} \sin \theta^{\prime} \cos \theta+\left(l m^{\prime}-l^{\prime} m\right) \sin \theta \sin \theta^{\prime}\right\} .
\end{aligned}
$$

This is the quaternion symbol of the rotation $A C$; its first term therefore expresses $\cos \mathrm{AC}$, which agrees with the fundamental theorem of spherical trigonometry; and the terms affected by $i, j, k$ are proportional to, and determine, the direction cosines of the positive axis of AC. The product $q q^{\prime}$ in like manner represents the rotation $C^{\prime} A^{\prime}$, if $A^{\prime}, C^{\prime}$ be points taken in $\mathrm{AB}, \mathrm{CB}$ produced, so that $\mathrm{BA}^{\prime}=\mathrm{AB}, \mathrm{BC}=\mathrm{CB}$.

The reader who is familiar with Sir W. Hamilton's researches on quatemions will observe that these and similar results, which are here primary, appear in his system of interpretation as secondary or polar; and the converse would easily be shown to be true also. (See particularly Irish Transactions, vol, xxi. part 2 , p. 80-86.) The reason of the difference is apparent. In Sir W. Hamilton's geometrical system, $i, j, k$ are not symbols of operation, but represent concrete units, namely, unit lines in fixed directions, so that $i x+j y+k z$ represents also, not an operation, but a concrete quantity, namely the radius vector of the point whose coordinates, referred to axes coinciding with the directions of $i, j, k$, are $x, y, z$. The quaternion $w+i x+j y+k z$ therefore represents what may be called a couple, or sum of two heterogeneous quantities, namely, an abstract length $w$, and a directed line $i x+j y+k z$. It is therefore not capable of a geometrical interpretation. This circumstance, however, as Sir W. Hamilton has abundantly shown, does not at all interfere with the use of quaternions in obtaining geometrical results.

In the system of interpretation which has been explained in this paper, every symbol is regarded as representing an operation; and every term of the quaternion, as well as the whole expression, has a geometrical interpretation. In fact, a qua- 
ternion expresses, in this system, what Sir W. Hamilton has called a geometrical quotient, or the operation which must be performed on one directed line to make it coincide in length and direction with another directed line. If a, $\mathrm{a}^{\prime}$ be two such lines, $l, m, n, l, m^{\prime}, n^{\prime}$ their direction cosines, and $\mu$ the arithmetical ratio of the length of a to that of $a^{\prime}$, then the geometrical quotient $\frac{a}{a^{\prime}}$ is expressed, as it is easy to see, by the quaternion

$$
q=\mu\left\{l l^{\prime}+m m^{\prime}+n n^{\prime}+i\left(m^{\prime} n-m n^{\prime}\right)+j\left(n^{\prime} l-n l^{\prime}\right)+k\left(l^{\prime} m-l m^{\prime}\right)\right\},
$$

and $\frac{a^{\prime}}{a}$ by another quaternion obtained from $q$ by writing $\mu^{-1}$ instead of $\mu$, and changing the signs of the three terms affected by $i, j, k$. According to this view, since $i, j, k$ are symbols of operation and not of quantity, there is nothing in the least strange in their not being commutative symbols, for we are familiar with non-commutative operations in other parts of analysis. I am not without a hope that this, with other considerations suggested by the preceding investigations, may tend to obviate any prejudice which may be felt against the theory of quaternions, as containing something arbitrary, mysterious, opposed to common notions, and incapable of interpretation. I trust it has sufficiently appeared that the theory may be completely divested of any such characteristics. At the same time $I$ am far from thinking that they really apply, in any sense implying an objection, to Sir W. Hamilton's mode of considering the subject. On the contrary, that mode possesses, in my estimation, besides its undoubted practical utility, the attraction of suggesting a novel and very interesting subject of inquiry; the properties, namely, of sets or sums of heterogeneous quantities. This, however, is a topic upon which I cannot here attempt to enter.

Oxford, May 18, 1850.

\section{On the Electricities of Steam. By Reuben Phillips, Esq.}

[Continued from p. 108.]

94. A COMMON laboratory brass tripod stand with A straight legs was insulated, by putting each leg into a glass tube previously closed at one end by the blowpipe; the glass tubes were securely jammed on by means of worsted, and a small piece of cork was placed in the bottom ot each tube, to prevent the metal bearing on the glass; the 